\title{
Twin embolisation syndrome-consequenses in the living fetus: A short review
}

Nikoli Aikaterini, Papalazarou Antigoni, Papachristodoulou Angeliki*, Nikolaos Spyridonos Ioakeimidis, Karachrysafi Sofia, Meditskou Soultana and Papamitsou Theodora

Laboratory of Histology and Embryology, Faculty of Medicine, Aristotle University of Thessaloniki, Aristotle University of Thessaloniki Campus, Greece

\begin{abstract}
Twin embolization syndrome (TES) is a rare complication of monozygotic monochorionic twining following in utero demise of the co-twin and is related to the vascular connections in monochorionic placentas. Passage of thromboplastic materials, thrombus, debris and toxins from the dying twin's circulation into the circulation of the surviving fetus results in ischemic structural defects of various systemic organs including the central nervous system (CNS). The purpose of this review is to analyse and present the consequenses of TES in the living fetus.
\end{abstract}

\section{Introduction}

Twin embolization syndrome (TES) is a rare complication of monozygotic monochorionic twining following in utero demise of the co-twin and is related to the vascular connections in monochorionic placentas. Passage of thromboplastic materials, thrombus, debris and toxins from the dying twin's circulation into the circulation of the surviving fetus results in ischemic structural defects of various systemic organs including the central nervous system (CNS) [1-3].

Monozygotic (MZ) or identical twins occur when a single ovum is fertilized to form one zygote which then divides into two separate embryos. By definition, monozygotic (MZ) twins carry an identical set of genetic information. About $1 / 3$ of twin pregnancies is monozygotic pregnancies. The frequency of monozygotic twinning is almost the same among different countries and is not affected by mother's age $[4,5]$. Monozygotic twinning starts by the stage of blastocyst at the end of the first week of pregnancy and is caused by the division of embryoblast in two embryonic structures. Eventually, two separate fetuses develop. So the two embryos, each one in a separate amniotic sac, grow into the same chorionic sac and share the same placenta, which is a monochorionic diamniotic placenta. In some cases, the primary separation of embryonic blastomeres causes monozygotic diamniotic dichorionic twins with two separate placentas [5].

Monozygotic twins occur in 3-5 per 1000 pregnancies. Monozygotic twins can be monochorionic or dichorionic. Approximately $75 \%$ of monozygotic twins are monochorionic. Only monochorionic twins are at risk for Twin to twin transfusion Syndrome. TTTS occurs in $5-38 \%$ of monochorionic twins. Thus, TTTS only occurs in same sex, monozygotic twins with monochorionic placentation. Twin embolization syndrome is the most severe complication of TTTS, so it is rarer than TTTS.

Twin embolization syndrome is thought to result from the passage of thromboplastic material, thrombus, debris and toxins into the circulation of the surviving twin, which leads to emboli, thrombosis, clots and circulatory problems, that cause ischemic structural defects in various organs (particularly the highly vascularised organs such as the central nervous system, gastrointestinal tract, and genitourinary system). The nature of damage to the surviving fetus appears to be related to the gestational age at the time of death of the co-twin (demise in early pregnancy results in atresia and tissue loss; demise later in pregnancy results in tissue infarction). These thromboplastic agents precipitate disseminated intravascular coagulation in the surviving fetus, with a hypercoagulable state due to a relative fetal antithrombin III deficiency. Acute haemodynamic shift from live to dead fetus resulting in hypoperfusion is more recently thought to play a role. In conclusion, anomalies of central nervous system and other systems appear in the surviving fetus and end up to twin embolization syndrome.

\section{Materials and methods}

Many medical papers have presented implications of TES in the surviving fetus as analysed below.

\section{Results}

\section{CNS anomalies}

\section{Fetal ventriculomegaly}

Fetal ventriculomegaly refers to the presence of dilated cerebral ventricles in utero. Fetal ventriculomegaly (ventricle width $>10 \mathrm{~mm}$ ) is an important finding in itself and it is also associated with other central nervous system abnormalities. The estimated prevalence may be at about $0.9 \%$ of all pregnancies, with a slightly increased male predilection [6].

${ }^{\star}$ Correspondence to: Papachristodoulou Angeliki, Medical Student, Laboratory of Histology and Embryology, Aristotle University of Thessaloniki, Aristotle University of Thessaloniki Campus, 54124 Thessaloníki, Greece, Tel: +306950135012; E-mail: angeliquep321@gmail.com

Key words: twin embolization syndrome, pregnancy, fetus

Received: June 04, 2018; Accepted: June 28, 2018; Published: July 02, 2018 
Fetal ventriculomegaly is defined as:

- $>10 \mathrm{~mm}$ across the atria of the posterior or anterior horn of lateral ventricles at any point in the gestation

- alternatively, a separation of more than $3 \mathrm{~mm}$ of the choroid plexus from the medial wall of the lateral ventricle may be used

The severity of ventriculomegaly can be further classified as:

- mild/borderline fetal ventriculomegaly: lateral ventricular diameter between 10-12 $\mathrm{mm}$

- moderate fetal ventriculomegaly: $12.1-15 \mathrm{~mm}$

- fetal ventriculomegaly (also sometimes classified as fetal hydrocephalus): severe lateral ventricular diameter $>15 \mathrm{~mm}$.

\section{Pathology}

Development of lateral ventricles differs depending on the trimester in which vetriculomegaly was created.

\section{First trimester}

The choroid plexus regularly fills the entire lateral ventricle, bilaterally.

\section{Second trimester}

- The choroid plexus begins to recede posteriorly but remains in close contact with the medial and lateral walls of the bodies and atria of the ventricles.

- Likewise, the lateral cerebral ventricle is large relative to the cerebral hemispheric width [7].

\section{Clinical presentation}

Infants with mild self-limited ventriculomegaly usually don't have any symptoms. If the ventriculomegaly is progressive the baby may show the signs and symptoms of hydrocephalus once it is born.

An infant with hydrocephalus may have:

- abnormally rapid head growth

- abnormally full fontanel

- distended scalp veins

- eyes that cannot look upward or appear to be staring downward (sunset sign)

- developmental delays

- irritability or abnormal sleepiness

- poor feeding

- vomiting

\section{Diagnosis}

The findings must be carefully evaluated, because of the association of fetal ventriculomegaly with other central nervous system anomalies. Pseudo-hydrocephalus as a possible diagnosis must be excluded and ultrasound is the screening modality of choice for initial evaluation. Fetal brain MRI may be useful too [7].

\section{Porencephaly}

Porencephaly is a rare congenital disorder that results in cystic degeneration and encephalomalacia and the formation of porencephalic cysts. The term is used variably among radiologists with its broadest definition being a cleft or cystic cavity within the brain, and its more narrow definition being a focal cystic area of encephalomalacia that communicates with the ventricular system and/or the subarachnoid space. Generally this term is used to describe any fluid-filled cavity in the fetal or neonatal brain [7].

\section{Pathology}

Porencephalic cysts are uncommon, but in some cases of TES severe porencephaly subsequently developed. The cysts are typically lined by white matter. They are thought to occur from focal encephalomalacia due to a localised cerebral insult most frequently during early gestation. Gliosis will develop if the insult is late enough, usually thought to be after the start of the third trimester, although perhaps insults as early as 20 weeks of gestation may result in gliosis [7].

\section{Clinical presentation}

Clinical manifestations are extremely variable. Patients may be asymptomatic or in other cases they develop spasticity, seizures, language impairment, mental retardation and motor deficits in the first year of life [6].

\section{Diagnosis}

Porencephalic cysts may appear as one or more intracranial cysts, on antenatal ultrasound scan, that communicate with the ventricular system and subarachnoid space. There may also be displacement of the midline ventricular echo because of asymmetrical ventricles [7].

The cysts' border is well-defined, on a CT scan, and occasionally enlarging cysts result in local mass effect [7]. On a MRI scan, the cyst is lined by white matter which may or may not demonstrate evidence of gliosis. Importantly the cyst is not lined by grey matter, helpful in distinguishing them from arachnoid cysts and schizencephaly [7]. Head circumference is variable. It may be normal or small, or alternatively synechiae can create a one-way valve effect with progressive enlargement of the cyst and skull expansion or hydrocephalus, resulting in an enlarged head.

\section{Fetal cerebral atrophy}

Fetal brain atrophy or shrinkage is rare and the cause is unknown in most cases. Among the most common risk factors is maternal alcohol consumption and intrauterine infection (Cytomegalovirus).

\section{Pathology}

Passage of thromboplastic materials, thrombus, debris and toxins from the dying twin's circulation into the circulation of the surviving fetus results in ischemic structural defects of various systemic organs including the central nervous system (CNS). Multiple lines of evidence support that cerebral ischemia is often the major factor that initiates cerebral injury in VLBW infants. In the near-term fetus, even brief severe global asphyxia, caused by a 10-minute occlusion of the umbilical cord, can result in prolonged hypoperfusion to cerebral gray and white matter. Systemic hypotension arising from intermittent or partial umbilical cord occlusion produced a variable degree of WMI in addition to primary damage to the cerebral cortex. Hence, cerebral hypoperfusion in conjunction with hypoxia appears to be a critical factor to generate significant preterm WMI. The spectrum of white matter pathology includes three major identifiable forms: focal cystic necrosis, focal microscopic necrosis and diffuse nonnecrotic lesions. 


\section{Clinical presentation}

The neonate can be small for gestational age, and can have bulging anterior and posterior fontanels, flat mid-face, smooth philtrum, hypertonia and increased deep tendon reflex in all extremities. The infant may have normal feeding tolerance, but can develop frequent neonatal seizures. 1 month after birth, the infant appears with suboptimal neurodevelopment and neurological signs of hypertonia, spastic tone, no eye contact and no social smile [9].

\section{Diagnosis}

On an ultrasound scan we may see decreased amniotic fluid and abnormal structures of the brain. Most of the space of the anterior fossa can be occupied by anechoic fluid or marked dilatation of the subarachnoid space around the brain, leading to a wide distance from the cranium to the brain surface. The cerebral hemispheres might display shrinkage to the center of the skull as a thin covering of the mid-brain. The thalami, brainstem, cerebelli and other structures in the posterior fossa can be well preserved and relatively normal in size.

On color-flow imaging, the circle of Willis can be visualized clearly and spectral Doppler ultrasonography may demonstrate a relatively low peak systolic velocity in the middle cerebral artery [9]. It is considered magnetic resonance imaging (MRI) of the fetal brain to be effective in confirming or denying diagnosis of fetal cerebral defects when ultrasonography was inconclusive or incomplete. Also, histological studies can confirm or deny ultrasonographic evidence [10].

\section{Cystic encephalomalacia}

The terms "Cystic encephalomalacia" is used to describe the presence of areas of necrosis that develop into cystic lesions inside the brain. These lesions are generally due to severe asphyxia and/or hypotension [11,12].

\section{Pathology}

Encephalomalacia, from the Greek "brain softening," refers to diffuse cerebral parenchymal volume loss. This typically occurs between the 20th and 30th week of gestation with the most common etiologies being maternal infection, trauma, or vascular insult. Impaired brain perfusion leads to neuronal injury and cell death. Neuronal cell loss occurs in two phases: primary and delayed. The high rates of cellular metabolism in the developing fetal brain make neurons particularly sensitive to ischemia and lead to primary neuronal cell loss within 30 minutes of onset of hypoxia and ischemia. However, many neurons are spared during the initial insult. Delayed loss results from free radical production. The cytotoxin-mediated apoptosis cascade usually occurs in the days following the insult. In contrast to porencephaly, or focal cystic white matter defects communicating with the ventricular system, encephalomalacia results in a global destruction of brain matter with symmetric bilateral effects. Because of neuronal cell death, diffuse brain atrophy results. In utero and postpartum outcomes are variable. However, this type of injury generally confers poor prognosis with neurodevelopmental delay, seizure disorders, and sensory and motor deficits [13].

\section{Clinical presentation}

The infant may have hypotonia during the first days of life. At 1 month of life the infant might still have hypotonia and delayed development and also it might be lethargic and irritable. At 3 months of life hypertonia and hyperreflexia are possible for the infant and it may appear still as irritable $[8,13]$.

\section{Diagnosis}

Fetal ultrasound can show lateral ventricular dilatation, white matter loss and extra-axial fluid. By fetal magnetic resonance imaging we can see enlargement of the lateral and third ventricles due to atrophy of surrounding white matter and deep gray matter. It is possible that cerebral sonograms show large cystic lesions in some regions of both or one of the cerebral hemispheres. A neonatal brain MRI can show the symmetry or asymmetry of cerebral white matter, diffuse, deep gray matter loss, microcephaly, ex vacuo hydrocephalus and hematomas. Doctors must also evaluate the placenta and the sepsis screen [13].

\section{Microcephaly}

Microcephaly is a medical condition in which the brain does not develop properly resulting in a smaller than normal head. Microcephaly may be present at birth or it may develop in the first few years of life. In fetal imaging it is usually defined as fetal head measurements (e.g. head circumference) falling under two standard deviations expected for gestation or falling under the 3rd percentile. The same definition is sometime applied to children and adults. Microcephaly is not a common condition. State birth defects tracking systems have estimated that microcephaly ranges from 2 babies per 10,000 live births to about 12 babies per 10,000 live births in the Unites States [14,15].

\section{Pathology}

Microcephaly can be divided into primary (or true) or secondary microcephaly. In primary microcephaly the brain never forms normally whereas in secondary microcephaly normal continued brain development is arrested by some defined insult / event. Microcephaly can be caused by aneuploidic and non aneuploidic syndromes, chromosomal and neurometabolic syndromes, utero infections, a substance's exposure in utero, such as drugs, alcohol or toxic chemicals, and other isolated features, such as hypotelorism and TES Syndrome, in which the passage of thromboplastic materials into the circulation of the surviving fetus leads to thrombosis, clots, circulatory problems and decreased perfusion of its brain. This ends up to delayed brain development or stop of brain's growing and microcephaly [14].

\section{Clinical presentation}

The first and more significant symptom is mental retardation. Apart from this, affected newborns have striking neurological defects and seizures. Additional signs are a smaller than normal head circumference that usually remains smaller than normal as the child grows, dwarfism or short stature, delayed motor and speech functions, facial distortions, hyperactivity, balance and coordination problems, and other brainrelated or neurological problems. Although some with the disorder may develop normal intelligence [14].

\section{Diagnosis}

Microcephaly can be diagnosed during pregnancy or after the baby is born. During pregnancy, microcephaly can sometimes be diagnosed with an ultrasound test. To see microcephaly during pregnancy, the ultrasound test should be done late in the 2nd trimester or early in the third trimester. To diagnose microcephaly after birth, a healthcare provider will measure the distance around a newborn baby's head, also called the head circumference, during a physical exam. The provider then compares this measurement to population standards by sex and age. Microcephaly is defined as a head circumference measurement that is smaller than a certain value for babies of the same age and sex. This measurement value for microcephaly is usually less than 2 standard 
deviations (SDs) below the average. The measurement value also may be designated as less than the $3 \mathrm{rd}$ percentile [15].

\section{Gastrointestinal anomalies}

\section{Gastroschisis}

Gastroschisis refers to extra-abdominal herniation (evisceration) of fetal or neonatal bowel loops (and occasionally portions or the stomach and or liver) into the amniotic cavity through a para-umbilical abdominal wall defect. The estimated incidence is at around 1-6 per 10,000 live births. There may be a male predilection and an increased incidence with younger maternal age.

\section{Pathology}

This condition occurs when an opening forms in the baby's abdominal wall. The baby's bowel pushes through this hole. The bowel then develops outside of the baby's body in the amniotic fluid. The passage of thromboplastic materials as it happens in TES Syndrome can be a cause, because this syndrome causes problems in vascular supply to the area in the abdominal wall adjacent to the umbilicus. This anomaly does not have a surrounding membrane (unlike an uncomplicated omphalocoele). It is the small bowel that herniates most often. The defect is invariably on the right side and usually measures between 2-4 $\mathrm{cm}$. There is no covering membrane or membrane remnant [16].

\section{Clinical presentation}

Half of malformations are considered related to the gastroschisis (intestinal atresia or stenosis, malrotation, cryptorchidism, amyoplasia, urinary tract obstruction). Other associated malformations occur which are not recognized to be secondary to the gastroschisis. Maternal serum alphafetoprotein is increased. Prominent among these are cardiac and limb defects. Fetal and neonatal mortality are increased, but neither appear related to lethal malformations [17].

\section{Diagnosis}

It is possible for gastroschisis to be detected in the third month of pregnancy. However, doctors most often perform evaluations for it at 20-24 weeks, after it has shown up on an ultrasound. It is most commonly diagnosed by ultrasound around weeks 18-20 of pregnancy.

An evaluation for gastroschisis consists:

- An ultrasound

- Possibly an MRI and/or a fetal echocardiogram to test baby's heart function

- Maternal serum alphafetoprotein measurement

- A meeting with a nurse, social worker and genetic counselor

- A team meeting with a maternal-fetal medicine specialist (MFM), pediatric surgeon and neonatologist

An important part of the evaluation is determining whether the condition is gastroschisis or omphalocele. These conditions can sometimes look similar on an ultrasound. In omphalocele, a sac from the umbilical cord covers and protects the intestines that are outside of the baby's body [16].

\section{Small bowel atresia}

Small bowel atresia can be ileal atresia, which is a congenital abnormality where there is significant stenosis or complete absence of a portion of the ileum, or jejunal atresia, which is a congenital anomaly characterised by obliteration of the lumen of the jejunum. The site of the atresia can be anywhere from the ligament of Treitz to the jejunoileal junction. There can be more than one atretic segment.

\section{Pathology}

Ileal atresia results from a vascular accident in utero that leads to decreased intestinal perfusion and subsequent ischemia a segment of bowel, or neonatal intestinal obstruction. As far as TES Syndrome is concerned, thromboplastic materials that pass to the circulation of the living fetus, cause clots and also decreased intestinal perfusion. This leads to narrowing, or in the most severe cases, complete obliteration of the intestinal lumen.

\section{Clinical presentation}

Common clinical characteristics of patients with jejunoileal atresia include the following:

- Polyhydramnios on antenatal ultrasonography (28\%)

- Prematurity (35\%)

- Low birth weight (25-50\%)

Classic signs include the following:

- Bilious emesis that warrants emergency surgical evaluation (most patients)

- Abdominal distention (in distal atresia)

- Jaundice (32\%)

- Failure to pass meconium in the first 24 hours (rule out Hirschsprung disease; passage of meconium does not rule out intestinal atresia)

Signs of continuous fluid loss include the following:

- Dehydration, manifested by sunken fontanel and dry membranes

- Decreased urine output (the best clinical indication of tissue perfusion)

- Tachycardia

- Decreased pulse pressure

- Low-grade fever

- Neurologic involvement, manifested by irritability, lethargy, or coma [18]

\section{Diagnosis}

Jejunoileal atresia can be identified on the basis of polyhydramnios present during prenatal ultrasonographic evaluation, bilious vomiting, abdominal distention, and jaundice. Some patients may not pass meconium in the first day of life.

Plain radiographs of the abdomen typically show the classic double-bubble sign: two distinct gas collections or air-fluid levels in the upper abdomen, resulting from the markedly dilated stomach and proximal duodenal bulb. There can be multiple dilated small bowel loops proximal to the atresia and the number of dilated loops increase as point of atresia becomes more distal.

In fluoroscopy, Contrast enema typically shows micro colon (small unused colon). 
Antenatal ultrasound may show:

- dilated proximal bowel loops, often greater than $7 \mathrm{~mm}$

- evidence of an in utero bowel perforation

- polyhydramnios, especially in cases where the atresia is proximal

In any case, small intestinal atresia must be differentiated from malrotation with midgut volvulus, total colonic Hirschsprung disease and meconium ileus [18].

\section{Urogenital Anomalies}

\section{Fetal renal cortical necrosis}

Renal cortical necrosis is a rare cause of acute renal failure secondary to ischemic necrosis of the renal cortex. Renal cortical necrosis is usually extensive, although focal and localized forms occur. In most cases, the medulla, juxtamedullary cortex, and a thin rim of subcapsular cortex are spared.

Renal cortical necrosis is classified into 5 pathologic forms, depending on severity, as follows:

1. Focal pathologic form - Kidneys show focally necrotic glomeruli without thrombosis and patchy necrosis of tubules

2. Minor pathologic form - Larger foci of necrosis are evident with vascular and glomerular thrombi

3. Patchy pathologic form - Patches of necrosis may occupy two thirds of the cortex

4. Gross pathologic form - Almost the entire cortex is involved; thrombosis of the arteries is more widespread

5. Confluent pathologic form - Kidneys show widespread glomerular and tubular necrosis with no arterial involvement

Renal cortical necrosis accounts more than $20 \%$ of acute renal failure during the third trimester of pregnancy. Renal cortical necrosis was detected by postmortem examination in $5 \%$ of infants aged 3 months or younger at death [19].

\section{Pathology}

Although the pathogenesis of the disease remains unclear, the presumed initiating factor is intense vasospasm of the small vessels. If this vasospasm is brief and vascular flow is reestablished, acute tubular necrosis results. More prolonged vasospasm can cause necrosis and thrombosis of the distal arterioles and glomeruli, and renal cortical necrosis ensues. In hemolytic-uremic syndrome (HUS) and septic abortion, an additional mechanism involves endotoxin-mediated endothelial damage that leads to vascular thrombosis. Studies have shown that patients with HUS with thrombotic microangiopathy (TMA) involving arteries have a higher likelihood of progressing into acute cortical necrosis compared with patients with predominant glomerular TMA. Renal cortical necrosis in placental abruption may be due to a combination of a hypercoagulable state, endothelial injury, and intravascular thrombosis.

In TES Syndrome, because of the passage of thromboplastic materials and toxins into the circulation of the surviving fetus, the perfusion to kidneys is low and this leads to ischemia and fetal renal cortical necrosis. Other causes of this condition are congenital heart disease, fetal-maternal transfusion, dehydration, perinatal asphyxia, anemia, placental hemorrhage, severe hemolytic disease and sepsis [18].

\section{Clinical presentation}

Kidney findings may include abdominal or bilateral costovertebral tenderness and palpable, tender kidneys.

Symptoms of Renal Cortical Necrosis (RCN)

- Flank Pain

- Hematuria

- Proteinuria

- Lower Urine Output

- Water Retention-

- Edema feet (swelling of feet)

- Enlarged liver

- Pleural effusion (fluid collection around lungs) $[19,20]$

Diagnosis

- Serum Electrolytes- High potassium level

- CBC- Low hemoglobin and red blood cell count

- Coagulation Panel- Blood clot disorder

- Urinalysis- Blood and protein in urine

- Ultrasound of the kidneys- Kidney size is smaller

- CT scan of the kidneys

- Kidney Biopsy- shows necrosis and blood clots [20]

\section{Respiratory Anomalies}

\section{Fetal hydrothorax}

A fetal hydrothorax refers to fluid in the fetal thoracic cavity. In many cases it represents a fetal pleural effusion. In selected cases it can be treated by in utero thoracocentesis or a formation of a in utero pleuro-amniotic shunt [7].

\section{Pathology}

The etiology of congenital hydrothorax is highly variable. Antenatal primary hydrothorax typically corresponds to congenital chylothorax, and is mainly due to lymphatic leakage because of a malformation, atresia or fistula, in the development of pulmonary lymphatic vessels of the thoracic duct. As far as TES Syndrome is concerned,the pulmonary lymphatic vessels have an obstruction or a malfunction due to passage of thromboplastic materials of dead fetus into the circulation of the surviving fetus. This leads to lymphatic leekage and chylothorax [21].

\section{Clinical presentation}

Primary fetal hydrothorax can resolve spontaneously in utero. However, there are specific signs that suggest the presence of an elevated intrathoracic pressure and therefore an increased risk for developing hydrops. Some of these signs are enlargement of the pleural effusion, evident mediastinal shift, and increased cardiac preload (abnormal ductus venosus blood flow, tricuspid regurgitation, and cardiomegaly). Also, hydramnio secondary to enlarged effusions causing esophageal compression and impeding fetal swallowing might lead to a poorer prognosis due to premature labor. The presence of any these markers, or the actual onset of hydrops, would be indications for urgent fetal intervention. 


\section{Diagnosis}

Prenatal diagnosis of congenital hydrothorax is based on the demonstration of fluid collection in the thorax, surrounding the lung uni or bilaterally, except for the hilium. It is usually diagnosed on the four-chamber view. If unilateral, it is considered as hypertensive if the intrathoracic pressure is high and the heart and all viscera are pushed into the contralateral hemithorax. If bilateral, it may produce increased venous return pressure and secondary hydrops $[1,2,7,11]$. Regarding the etiologic diagnosis, an initial thoracocentesis is useful for diagnosing congenital chylothorax (more than $80 \%$ of lymphocytes in the blood count) and for decompressing the lungs $[12,13]$. Usually, this procedure alone will not resolve the problem, since in more than $70-80 \%$ of the cases the liquid will reaccumulate quickly.

Differential diagnosis with pericardial effusion is usually easy and straightforward, whenever the scan is performed by a medium experienced examiner. In pericardial effusion the fluid is surrounding the heart and both lungs remain attached to the posterior wall of the chest [21].

\section{Diagnosis}

Prenatal: Sonographic evaluation of the fetus is commonly performed during pregnancy in order to diagnose any abnormalities that may occur through the first, second or third semester of it. As far as monochorionic twin pregnancies are corcerned, sonography is a method capable of diagnosing demise of one of the twins in utero ( vanishing twin syndrome), with or without associated bleeding referred from the mother [8]. The presence of a dead twin combined with a surviving twin with various anomalies may suggest the diagnosis of the twin embolisation syndrome. Sonographic evaluation of the surviving twin may diagnose CNS anomalies such as fetal ventriculomegaly, porencephaly, fetal cerebral atrophy, custic encephalomalacia and microcephaly. Non CNS anomalies such as small bowel atresia, fetal hydrothorax, gastroschisis, aplasia cutis, hydrothorax and renal cortical necrosis may also be diagnosed through sonography. Twin embolisation syndrome is not usually diagnosed in cases that the death of the co-twin happened during the first semester of the pregnancy $[10,11]$.

Another method that is used to diagnose twin embolisation syndrome is Doppler ultrasound. Doppler assessment of the placental circulation plays an important role in screening for impaired placentation and its complications of pre-eclampsia, intrauterine growth restriction and perinatal death. Assessment of fetal circulation is essential in understanding the pathophysiology of a wide range of pathological pregnancies and their clinical management. In Doppler ultrasound scanners, a series of pulses is transmitted to detect movement of blood. It is proved that color Doppler ultrasound sonography can play an important role in identificating the communicating placental vessels in pregnancies with twin-to-twin transfusion syndrome or its severe form, the twin embolization syndrome [12].

Postnatal: After birth, the surviving twin who has CNS and nonCNS anomalies, which are referred in previous paragraphs, has to be put through some more diagnostic examinations. Magnetic resonance imaging (MRI) is a powerful and versatile diagnostic tool. Applications of MR technology are rapidly expanding for all patient populations, including infants receiving newborn intensive care. The surviving infant now routinely undergoes MRI of the head, chest, abdomen, spine and pelvis for a multitude of diagnostic purposes. MRI techniques are superior to most other diagnostic and prognostic neuroimaging applications. X-rays or computed tomography(CT) scans can also be used but, unlike MRI, they use ionizing radiation. Instead, MRI uses an extremely powerful static magnetic field, rapidly changing gradient magnetic fields, and radiofrequency electromagnetic impulses to produce detailed anatomic or functional images of the brain and other soft tissues of the body of the surviving twin [14].

After the demise of one of the twins during pregnancy, maternal and fetal monitoring is performed until birth in order to prevent complications in the surviving twin.

\section{Maternal monitoring includes:}

- Routine antenatal investigations ( ABO Rh, Hb, GCT, Urine routine and microscopy and viral markers)

- Weekly coagulation profile (platelet count, PT aPTT, BT and CT)

- FDP and D-dimer

Fetal monitoring includes:

- Daily fetal movement count

- Biweekly NST in pregnancies more than 32 weeks

- Biweekly USG with biophysical profile and colour Doppler [13]

Theoretically, premature delivery and treatment of the surviving fetus at an early stage may reduce the degree of cerebral as well as other organ damage. The benefits of this theoretical approach must be balanced by the inevitable risks of prematurity, complications of anticoagulant therapy and risks after treatment such as fetoscopic laser vessel occlusion [2].

\section{Discussion}

In cases of severe twin- to- twin transfusion syndrome, may not only one but both of the co- twins die prenatally. The most severe form of twin- to- twin transfusion syndrome with acute hydramnios and stuck twin usually occurs between 18 and 26 weeks gestation, with a survival rate of $20-45 \%$ for those identified before 28 weeks. Passage of thromboplastic- like material or embolic debris to the circulation of the surviving twin may cause multiple organ ischemia which has poor prognosis [2]. Apart from this, the surviving twin is possible to face premature birth (35.1 -38.2 week) or very premature birth (<32 week) which increases the risk of mortality. Low birth weight is also linked to twin embolisation syndrome and affects the prognosis [15].

The surviving twin will also have associated with the twin embolisation syndrome congenital anomalies which will affect prognosis. Three common anomalies are ventriculomegaly, porencephaly and microcephaly. Cases of ventriculomegaly resolved, regressed or remained stable in utero will exhibited good prognosis (85\% of all cases). Ventriculomegaly with an transverse atrial size $>=12$ $\mathrm{mm}$ or progression in utero are usually associated with a poor prognosis, which should be observed carefully [16]. As for porencephaly, the extend of impairment is somewhat related to the size and location of the lesion [17]. Microcephaly generally has good prognosis but can lead to disability [18].

When the diagnosis of an intrauterine fetal death, in the setting of a monochorionic gestation, is made near term, immediate delivery seems appropriate to improve the prognosis of the surviving twin. Earlier in pregnancy, in severe previable twin embolisation syndrome, fetoscopic laser occlusion of placental vessels or cord occlusion of the dead twin was reported to prevent further damage to the surviving twin. Meticulous sonographic evaluation permits counseling for the appropriate treatment, prognosis and risks in twin embolisation syndrome [2]. 


\section{Case studies}

A 19 year old primigravida was referred for sonographic evaluation at 14 weeks of her first, spontaneous pregnancy. Vaginal sonography revealed a twin pregnancy. One fetus had no heartbeat, while the other fetus had a normal sonographic examination. Both fetuses had CRL and BPD measurements appropriate for gestational age. A thin dividing membrane was clearly seen, revealing a monochorionic, diamniotic twin pregnancy with missed twin.

Owning to religious reservations, the triple test wasn't performed. At 22 weeks gestation the patient was referred for another sonographic examination. The surviving fetus was found to have bilateral ventriculomegaly $(13 \mathrm{~mm})$. The third and fourth ventricles were normal. The nuchal fold, heart, skin and thorax were found to be normal. Bowel distension suggesting obstruction was observed with multiple intraabdominal calcifications. AC, $\mathrm{HC}$ and $\mathrm{FC}$ had dimensions compatible with a gestation of two weeks earlier, with a normal amount of amniotic fluid suggesting early intrauterine growth retardation. The second fetus, of a size compatible with a gestation of 12 to 13 weeks, was clearly in an individual sac with no pulse, located above the cervix. Cordogenesis or amniocentesis was denied by the couple for religious reasons.

At 30 weeks gestation sonographic evaluation of the fetal brain revealed right- sided ventriculomegaly of $11.4 \mathrm{~mm}$ and left-sided unilateral porencephaly. The size of the penis was small. BDL, FL and AC were compatible to 28 weeks and the estimated fetal weight was $1210 \mathrm{~g}$. The second, missed twin, had a CRL of $45 \mathrm{~mm}$. The result of Doppler flow studies performed on the umbilical artery and the carotid artery of the surviving twin were normal.

At 35 weeks gestation the patient experienced premature contractions and minimal vaginal bleeding. Clinical examination revealed that the uterus was distended, suggesting polyhydramnios, with no cervical dilatation. Sonographic examination verified severe polyhydramnios. Asymetrical hydrocephalus, with porencephaly of the right cortex and left posterior cortex, was present, and the fetal abdominal sonography revealed bowel dilatation with multiple calcifications. A cyst measuring $15 \mathrm{~mm}$ in diameter was found in the spleen, probably secondary to an infarct.

At 36 weeks gestation the patient had a normal delivery of a boy with Apgar scores of 5 and 9 at 1 and $5 \mathrm{~min}$. After tracheal suction the neonate had oxygen saturation of $94 \%$ in room air, pulse rate of 148 beats $/ \mathrm{min}$ and blood pressure of $60 / 37 \mathrm{~mm} \mathrm{Hg}$.

Placental examination revealed a three-vessel cord and a dividing membrane compatible with monochorionic diamniotic twins. The second twin was found attached to the dividing membrane with a short cord. However, pathological confirmation of vascular connections between the two sides of the placenta was impossible because of necrosis of the second twin placenta due to the relatively long period between fetal death and the delivery.

The neonate had a symmetric head with an HC of $31.6 \mathrm{~m}$ and clear signs of aplasia cutis congenital mainly on the lower extremities. Although s mild general hypotonic appearance was evident, symmetric movements of the upper and lower limbs were noticed. Examination of the external genitalia revealed a micropenis with both testes in the scrotum. An abdominal radiograph showed a triple bubble, consistent with jejuna atresia. An EEG revealed a minimal function, suggesting serious extended structural damage and a head scan CT revealed severe bilateral hydrocephalus due to progressive porencephaly with minimal brain tissue. The karyotype was normal $(46 \mathrm{X}, \mathrm{Y})$. Parenteral nutrition was started and a nasogastric tube was used to drain excessive secretions. However, the infant died after 2 months owing to aspiration pneumonia. Autopsy was denied by the parents for religious reasons [2].

\section{References}

1. Kawamura T, Morioka T, Fukui K, Nishio S, Hikino S, et al. (2002) [Symmetrical hemispheric porencephalic cysts due to twin embolization syndrome: a case report]. No To Shinkei 54: 723-728. [Crossref]

2. Elchalal U, Tanos V, Bar-Oz B, Nadjari M (1997) Early second trimester twin embolization syndrome. J Ultrasound Med 16: 509-512. [Crossref]

3. Gray P, Rouse GA, de Lange M (1993) Sonographic Evaluation of Twin Embolization Syndrome.A Report of Three Cases and Review of the Literature. J Diagnostic Med Sonology 9: 3-10

4. Moore KL, Persaud TVN (2013) The Developing Human, Clinically Oriented Embryology, 8th Edition.

5. Zhang R, Thiele H, Bartmann P, Hilger AC, Berg C, et al. (2016) Whole-Exome Sequencing in Nine Monozygotic Discordant Twins. Twin Res Hum Genet 19: 60-65. [Crossref]

6. Ventriculomegaly Symptoms \& Causes. http://www.childrenshospital.org/conditionsand-treatments/conditions/v/ventriculomegaly/symptoms-and-causes

7. Twin embolisation syndrome. https://radiopaedia.org/articles/twin-embolisationsyndrome

8. Caballero P, Del Campo L, Ocón E (1991) Cystic encephalomalacia in twin embolization syndrome. Radiology 178: 892-893. [Crossref]

9. Tongsong T, Puntachai P, Mekjarasnapha M, Traisrisilp K (2014) Severe fetal brain shrinkage following heavy maternal alcohol consumption. Ultrasound Obstet Gynecol 44: 245-247. [Crossref]

10. D'Ercole C, Girard N, Boubli L, Potier A, Chagnon C, et al. (1993) Prenatal diagnosis of fetal cerebral abnormalities by ultrasonography and magnetic resonance imaging. Eur J Obstet Gynecol Reprod Biol 50: 177-184. [Crossref]

11. Back SA (2014) Cerebral white and gray matter injury in newborns: new insights into pathophysiology and management. Clin Perinatol 41: 1-24. [Crossref]

12. Frigieri G, Guidi B, Costa Zaccarelli S, Rossi C, Muratori G, et al. (1996) Multicystic encephalomalacia in term infants. Childs Nerv Syst 12: 759-764. [Crossref]

13. Love R, Lee A, Matiasek A, Carter W, Ylagan M (2014) Prenatal Diagnosis of Feta Encephalomalacia after Maternal Diabetic Ketoacidosis. AJP Rep 4: e97-e100. [Crossref]

14. Charles Patrick Davis. Microcephaly facts, www.mediciNet.com

15. Centers for Disease Control and Prevention (2013) Major birth defects data from population-based birth defects surveillance programs in the United States, 2006-2010. Send to

16. Birth Defects Res A Clin Mol Teratol 97:S1-S172.

17. Gastroschisis, www.CincinnatiChildrens.org

18. Hunter AG, Stevenson RE (2008) Gastroschisis: clinical presentation and associations. Am J Med Genet C Semin Med Genet 148C: 219-230. [Crossref]

19. Jaime Shalkow (2017) Small Intestinal Atresia and Stenosis Clinical Presentation. www.Medscape.com

20. Prasad Devarajan (2015) Renal Cortical Necrosis Differential Diagnoses. www. Medscape.com

21. Pramod Kerkar (2018) Renal Cortical Necrosis (RCN): Types, Causes, Risk Factors, Symptoms, Diagnosis, Treatment.

22. Twin Embolisation Syndrome. www.Visuog.org

Copyright: (C)2018 Aikaterini N. This is an open-access article distributed under the terms of the Creative Commons Attribution License, which permits unrestricted use, distribution, and reproduction in any medium, provided the original author and source are credited. 